\title{
Possible link between the power spectrum of interstellar filaments and the origin of the prestellar core mass function ${ }^{\star}$
}

\author{
A. Roy ${ }^{1}$, Ph. André ${ }^{1}$, D. Arzoumanian ${ }^{1,2}$, N. Peretto ${ }^{3}$, P. Palmeirim ${ }^{1}$, V. Könyves ${ }^{1}$, N. Schneider ${ }^{1,4}$, M. Benedettini ${ }^{5}$, \\ J. Di Francesco ${ }^{6,7}$, D. Elia ${ }^{5}$, T. Hill ${ }^{1,8}$, B. Ladjelate $^{1}$, F. Louvet ${ }^{9}$, F. Motte ${ }^{1}$, S. Pezzuto ${ }^{5}$, E. Schisano ${ }^{5}$, Y. Shimajiri ${ }^{\text {, }}$, \\ L. Spinoglio ${ }^{5}$, D. Ward-Thompson ${ }^{10}$, and G. White ${ }^{11,12}$
}

${ }^{1}$ Laboratoire AIM, CEA/DSM-CNRS-Université Paris Diderot, IRFU/Service d'Astrophysique, C.E. Saclay, Orme des Merisiers, 91191 Gif-sur-Yvette, France e-mail: [Arabindo.Roy; philippe.andre]@cea.fr

2 Institut d'Astrophysique Spatiale, CNRS/Université Paris-Sud 11, 91405 Orsay, France

3 School of Physics \& Astronomy, Cardiff University, Cardiff, CF29, 3AA, UK

${ }^{4}$ Université de Bordeaux, LAB, CNRS/INSU, UMR 5804, BP 89, 33271 Floirac Cedex, France

5 INAF-Istituto di Astrofisica e Planetologia Spaziali, via Fosso del Cavaliere 100, 00133 Rome, Italy

6 Department of Physics and Astronomy, University of Victoria, PO Box 355, STN CSC, Victoria, BC, V8W 3P6, Canada

National Research Council Canada, 5071 West Saanich Road, Victoria, BC, V9E 2E7, Canada

8 Joint ALMA Observatory, Alonso de Córdova 3107, Vitacura, Santiago, Chile

9 Departamento de Astronomía, Universidad de Chile, 1058 Santiago, Chile

10 Jeremiah Horrocks Institute, University of Central Lancashire, Preston, Lancashire, PR1 2HE, UK

11 Department of Physics and Astronomy, The Open University, Walton Hall Milton Keynes, MK7 6AA, UK

12 RAL Space, STFC Rutherford Appleton Laboratory, Chilton Didcot, Oxfordshire OX11 0QX, UK

Received 28 April 2015 / Accepted 8 September 2015

\section{ABSTRACT}

\begin{abstract}
A complete understanding of the origin of the prestellar core mass function (CMF) is crucial. Two major features of the prestellar $\mathrm{CMF}$ are 1) a broad peak below $1 M_{\odot}$, presumably corresponding to a mean gravitational fragmentation scale, and 2) a characteristic power-law slope, very similar to the Salpeter slope of the stellar initial mass function (IMF) at the high-mass end. While recent Herschel observations have shown that the peak of the prestellar CMF is close to the thermal Jeans mass in marginally supercritical filaments, the origin of the power-law tail of the CMF/IMF at the high-mass end is less clear. In 2001, Inutsuka proposed a theoretical scenario in which the origin of the power-law tail can be understood as resulting from the growth of an initial spectrum of density perturbations seeded along the long axis of star-forming filaments by interstellar turbulence. Here, we report the statistical properties of the line-mass fluctuations of filaments in the Pipe, Taurus, and IC 5146 molecular clouds observed with Herschel for a sample of subcritical or marginally supercritical filaments using a 1D power spectrum analysis. The observed filament power spectra were fitted by a power-law function $\left(P_{\text {true }}(s) \propto s^{\alpha}\right)$ after removing the effect of beam convolution at small scales. A Gaussian-like distribution of power-spectrum slopes was found, centered at $\bar{\alpha}_{\text {corr }}=-1.6 \pm 0.3$. The characteristic index of the observed power spectra is close to that of the 1D velocity power spectrum generated by subsonic Kolomogorov turbulence $(-1.67)$. Given the errors, the measured powerspectrum slope is also marginally consistent with the power spectrum index of -2 expected for supersonic compressible turbulence. With such a power spectrum of initial line-mass fluctuations, Inutsuka's model would yield a mass function of collapsed objects along filaments approaching $\mathrm{d} N / \mathrm{d} M \propto M^{-2.3 \pm 0.1}$ at the high-mass end (very close to the Salpeter power law) after a few free-fall times. An empirical correlation, $P^{0.5}\left(s_{0}\right) \propto\left\langle N_{\mathrm{H}_{2}}\right\rangle^{1.4 \pm 0.1}$, was also found between the amplitude of each filament power spectrum $P\left(s_{0}\right)$ and the mean column density along the filament $\left\langle N_{\mathrm{H}_{2}}\right\rangle$. Finally, the dispersion of line-mass fluctuations along each filament $\sigma_{M_{\text {line }}}$ was found to scale with the physical length $L$ of the filament roughly as $\sigma_{M_{\text {line }}} \propto L^{0.7}$. Overall, our results are consistent with the suggestion that the bulk of the CMF/IMF results from the gravitational fragmentation of filaments.
\end{abstract}

Key words. ISM: structure - stars: formation - stars: luminosity function, mass function - evolution

\section{Introduction}

Understanding the origin of the stellar initial mass function (IMF) is a fundamental open problem in modern astrophysics (see, e.g., Offner et al. 2014 for a recent review). Since the end of the 1990s, several observational studies of prestellar dense cores in nearby molecular clouds have found a strong link between the prestellar core mass function $(\mathrm{CMF})$ and the IMF (e.g., see Motte et al. 1998; Alves et al. 2007; Könyves et al. 2010, 2015), suggesting that the IMF is at least partly the result

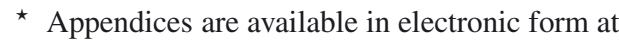
http://www . aanda.org of the core formation process. Theories of the CMF/IMF based on gravo-turbulent fragmentation (e.g., Padoan \& Nordlund 2002; Hennebelle \& Chabrier 2008, Hopkins 2012) are consistent with this view, but do not account for the fact that most cores/stars appear to form within interstellar filaments. Indeed, recent Herschel observations (e.g., André et al. 2010; Molinari et al. 2010; Arzoumanian et al. 2011; Hill et al. 2011; Könyves et al. 2015) emphasize the role of interstellar filaments in starforming clouds and support a paradigm for star formation in which the formation of $\sim 0.1 \mathrm{pc}$-wide filaments and the subsequent fragmentation of the densest filaments into prestellar cores are two key steps in the star formation process (see André et al. 2014). The results of the Herschel Gould Belt survey (HGBS) 
further suggest that gravitational fragmentation of marginally supercritical filaments with masses per unit length $M_{\text {line }}$ approaching the critical line mass of nearly isothermal $\sim 10 \mathrm{~K}$ gas cylinders $M_{\text {line,crit }}=2 c_{\mathrm{s}}^{2} / G \sim 16 M_{\odot} /$ pc (Ostriker 1964; Inutsuka \& Miyama 1997) may be responsible for the peak of the prestellar $\mathrm{CMF}$ at $\sim 0.6 M_{\odot}$, as observed in the Aquila cloud complex for example (André et al. 2014; Könyves et al. 2015). This idea is consistent with the view that the peak of the IMF is related to the typical Jeans mass in star-forming clouds (Larson 1985).

In gravo-turbulent fragmentation theories of the CMF/IMF, the peak of the IMF results from a combination of thermal physics, setting the mean thermal Jeans mass in the parent cloud, and turbulence effects, through the turbulence Mach number (Hennebelle \& Chabrier 2008; Hopkins 2012; Chabrier et al. 2014). In the filamentary picture proposed by André et al. (2014), a characteristic thermal Jeans mass results from the existence of a critical line mass for filaments (which depends only on gas temperature) and from the characteristic filament width $\sim 0.1 \mathrm{pc}$ measured with Herschel (Arzoumanian et al. 2011), which is close to the sonic scale of turbulence (Federrath et al. 2010, and references therein) in low-density molecular gas. Taken together, this sets a density or column density threshold for prestellar cores and star formation as observed in nearby clouds with Herschel (André et al. 2010; Könyves et al. 2015) and Spitzer (Heiderman et al. 2010; Lada et al. 2010), respectively.

Although the thermal Jeans mass within marginally supercritical filaments may account for the peak of the prestellar $\mathrm{CMF}$, pure thermal fragmentation of filaments at the threshold of gravitational instability is expected to lead to a sharply peaked CMF (such as a delta function) and can hardly explain the Salpeter power-law regime of the IMF at the high-mass end. Inutsuka (2001) suggested, however, that a Salpeter-like CMF can quickly develop within filaments provided that turbulence has generated the appropriate power spectrum of initial density fluctuations in the first place. More specifically, Inutsuka (2001) found that the hierarchical structural property of the perturbed density field along filaments may statistically produce a population of cores with a power-law mass function. Inutsuka's approach consisted of counting the distribution of "isolated" collapsed regions of mass scale $M$ above a critical density using the Press-Schechter formalism (Press \& Schechter 1974; Jedamzik 1995), where the statistics of such regions depend on the logarithmic slope of the power spectrum characterizing the initial fluctuating density field. Inutsuka (2001) showed that the resulting core mass distribution converges toward a Salpeter-like mass function $\mathrm{d} N / \mathrm{d} M \propto M^{-2.5}$ when the power spectrum of the field of initial line-mass fluctuations approaches $P(s) \propto s^{-1.5}$. This value was adopted on a purely ad hoc basis, however, and the true statistical nature of the density fluctuations along filaments remained to be quantified observationally. Another important assumption of Inutsuka's model was that the initial line-mass fluctuations along filaments could be represented by a Gaussian random distribution.

In this paper, we exploit the unprecedented sensitivity and resolution of Herschel submillimeter continuum images as well as the proximity of the molecular clouds targeted as part of the HGBS key project to characterize, for the first time, the statistical properties of the line-mass fluctuations along interstellar filaments by analyzing their 1D power spectrum. Our main goal is to constrain observationally the validity of the theoretical model outlined by Inutsuka (2001) for the origin of a Salpeterlike prestellar CMF above $\sim 1 M_{\odot}$.

\section{Herschel observations and column density maps}

The three target fields that we used in the present analysis were all observed with the Herschel ${ }^{1}$ space observatory (Pilbratt et al. 2010) as part of the HGBS key project (André et al. 2010); they cover surface areas of $\sim 1.5 \times 1.5, \sim 6^{\circ} \times 2.5$, and $\sim 1^{\circ} .6 \times 1.6$ in the IC 5146, Pipe, and Taurus molecular clouds, respectively (Arzoumanian et al. 2011; Peretto et al. 2012; Palmeirim et al. 2013). These three regions were mapped at a scanning speed of $60^{\prime \prime} \mathrm{s}^{-1}$ in parallel mode simultaneously at five Herschel wavelengths using the SPIRE (Griffin et al. 2010) and PACS (Poglitsch et al. 2010) photometric cameras. The data were reduced using HIPE version 7.0. For the SPIRE data reduction, we used modified pipeline scripts. Observations during the turnaround of the telescope were included, and a destriper module with a zero-order polynomial baseline was applied. The default naïve mapper was used to produce the final maps. For the PACS data, we applied the standard HIPE data reduction pipeline up to level 1, with improved calibration. Further processing of the data, such as subtraction of thermal and nonthermal low-frequency noise and map projection was performed with Scanamorphos v11 (Roussel 2013). The Scannamorphos map-maker avoids any high-pass filtering, which is crucial for preserving extended emission. We adjusted the zero-point values of individual Herschel images based on cross-correlations of the Herschel data with IRAS and Planck data (cf. Bernard et al. 2010). Figure 1 shows an example of $250 \mu \mathrm{m}$ surface brightness image of the Pipe Nebula at the native resolution of $18^{\prime \prime} .2$.

For the present analysis, we needed high-resolution column density maps to recover small-scale fluctuations which would otherwise be smeared due to beam convolution. We employed two methods for generating such column density maps. First, we generated them at an effective resolution corresponding to the SPIRE $250 \mu \mathrm{m}$ beam following the method described in Appendix A of Palmeirim et al. (2013). In this method, largescale information is obtained from a standard Herschel column density map constructed at the SPIRE $500 \mu \mathrm{m}$ resolution, while finer details are recovered by constructing more approximate column density maps at the resolution of SPIRE $350 \mu \mathrm{m}$ and SPIRE $250 \mu \mathrm{m}$ data. Following the steps described in Palmeirim et al., we fitted a single-temperature modified blackbody to the observed data on a pixel-by-pixel basis, adopting a wavelengthdependent dust opacity of the form $\kappa_{\lambda}=0.1(\lambda / 300 \mu \mathrm{m})^{-\beta} \mathrm{cm}^{2}$ per $\mathrm{g}$ (of gas + dust) with an emissivity index $\beta=2$ (cf. Hildebrand 1983; Roy et al. 2014). In the second method, we directly converted the surface brightness maps observed at $250 \mu \mathrm{m}$ $I_{250}$ into approximate column density maps by using the relation $N_{\mathrm{H}_{2}}=I_{250} /\left(B_{250}\left[T_{\mathrm{d}, \mathrm{SED}}\right] \kappa_{250} \mu_{\mathrm{H}_{2}} m_{\mathrm{H}}\right)$, where $\mu_{\mathrm{H}_{2}}=2.8$ is the mean molecular weight and $T_{\mathrm{d}, \mathrm{SED}}$ is the spectral energy distribution (SED) dust temperature obtained by fitting a modified blackbody to the SED observed between $160 \mu \mathrm{m}$ and $500 \mu \mathrm{m}$ with Herschel data toward each line of sight. For the Pipe Nebula filament shown in Fig. 2, for instance, the median dust temperature along the filament crest is $\bar{T}_{\mathrm{d}, \mathrm{SED}}=13.5 \mathrm{~K}$. The advantage of the first method is that it produces a more accurate column density map at the $18.2^{\prime \prime}$ resolution of the SPIRE $250 \mu \mathrm{m}$ data. The disadvantage, however, is that the resulting map is significantly noisier than the SPIRE $250 \mu \mathrm{m}$ map and the point spread function (PSF) is more difficult to characterize because of the various steps involved in the processing. In contrast, in the second method (which simply produces modified $250 \mu \mathrm{m}$ maps),

1 Herschel is an ESA space observatory with science instruments provided by European-led Principal Investigator consortia and with important participation from NASA. 


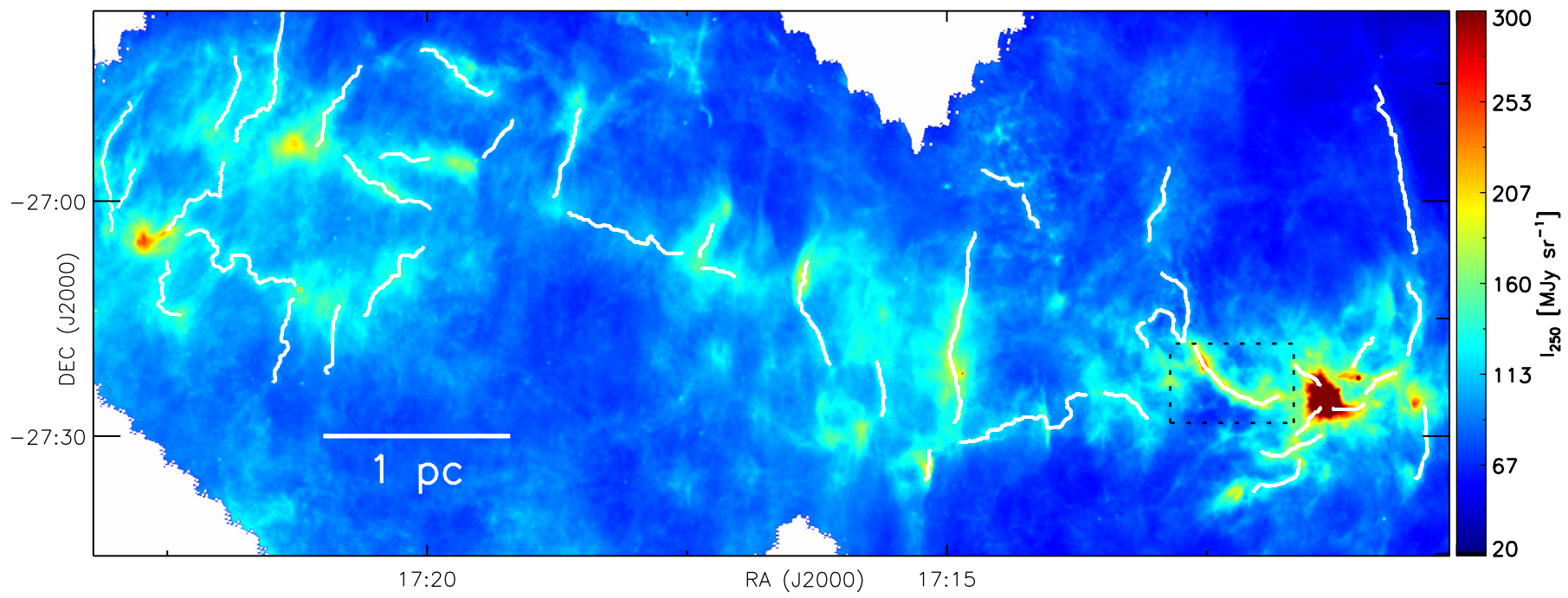

Fig. 1. Herschel $250 \mu \mathrm{m}$ surface brightness image of the Pipe molecular cloud observed as a part of the HGBS key project (cf. Peretto et al. 2012). The image is displayed at the diffraction limited $250 \mu \mathrm{m}$ beam resolution of 18.'2. The 43 filaments traced with the DisPerSE algorithm (Sousbie et al. 2011) are highlighted in white. A more detailed view of the filament enclosed by the black dashed rectangle is shown in Fig. 2. (This figure is available in color in electronic form.)

the effective PSF is exactly the same as that of the SPIRE $250 \mu \mathrm{m}$ photometer. Since an accurate knowledge of the beam is important in the present power-spectrum study, we report results based on the second method in the body of the paper. The results of a power-spectrum analysis performed using high-resolution column density maps produced with the first method are given in Appendix B. They confirm the robustness of our conclusions.

\section{Filament identification}

For the purpose of detecting coherently elongated filamentary structures in the column density maps as well as the modified $250 \mu \mathrm{m}$ images, we employed the DisPerSE algorithm which traces the crests of segments of topological filaments by connecting saddle points to maxima following the gradient in an image (Sousbie et al. 2011; see also Arzoumanian et al. 2011; Hill et al. 2011 for details on practical applications of DisPerSE on Herschel images). It is important to point out here that DisPerSE was used solely for tracing the filament crests, while the filament longitudinal profiles were measured directly on the original images. Our filament sample consists of 28 filaments in the Taurus cloud (adopted distance $d=140 \mathrm{pc}$; Elias 1978), 42 filaments in the Pipe nebula region $(d=145 \mathrm{pc}$; Alves et al. 2007), and 36 filaments in the IC 5146 cloud ( $d=460 \mathrm{pc}$; Lada et al. 1999). Combining the three regions, we identified a total of 106 filaments of various lengths above a $5 \sigma$ persistence level of $\sim 10^{21} \mathrm{~cm}^{-2}$; however, for a proper power-spectrum analysis, which was our ultimate goal, we had to select moderately long filaments to have an adequate number of Fourier modes at small spatial frequencies (see Sect. 5 below). Our final analysis was thus based on the subset of 80 filaments that are longer than 5.5 ( $\sim 18$ beams).

\section{Column density/line mass fluctuations along selected filaments}

Figure 2 shows an example of a longitudinal profile of column density or line-mass fluctuations along a marginally critical filament in the Pipe molecular cloud. As the mass per unit length or line mass is a fundamental variable in cylindrical geometry (cf. Inutsuka 2001), we converted the observed column density fluctuations to line-mass fluctuations by making use of the quasiuniversal filament width $\sim 0.1 \mathrm{pc}$ found by Arzoumanian et al. (2011). The line-mass fluctuations corresponding to the example filament in Fig. 2a are shown in Fig. 2b, while the equivalent column density fluctuations are quantified on the right ordinate axis of Fig. 2b. To estimate fluctuations intrinsic to the selected filaments themselves, a mean line-of-sight background column density was subtracted from the observed data for each field. The dotted horizontal line in Fig. $2 b$ shows the mean line-mass of the filament $\left(\sim 15.5 M_{\odot} / \mathrm{pc}\right)$, and allows the relative strength of the line-mass fluctuations about the mean to be evaluated. In this example, and for all other subcritical filaments in our sample, the line-mass fluctuations are small in relative amplitude, i.e., $\frac{\mid M_{\text {line }}-\left\langle M_{\text {line }}\right\rangle}{\left\langle M_{\text {line }}\right\rangle}<0.1$, implying that the perturbation modes are in the linear regime (see also Sect. 6 below).

Figure 3 shows the distribution of normalized line-mass fluctuations, $\delta=\frac{M_{\text {line }}-\left\langle M_{\text {line }}\right\rangle}{\left\langle M_{\text {line }}\right\rangle}$, along the 67 filaments in our sample that are subcritical over their entire length. It can be seen in Fig. 3 that the distribution of line-mass fluctuations for subcritical filaments is nearly Gaussian, which is an important assumption of the CMF/IMF model calculations presented by Inutsuka (2001). It can also be seen that the density/line-mass fluctuations along subcritical filaments are typically less than $10 \%$, i.e., in the linear regime.

The power spectrum of the fluctuations observed along the filament in Fig. 2 is shown in Fig. 4 and discussed in Sect. 5 below. Panels a and b of Figs. 5 and 6 are similar to Fig. 2, but show two extreme examples of a subcritical filament (with $\left.\left\langle M_{\text {line }}\right\rangle \sim 3 M_{\odot} / \mathrm{pc} \ll M_{\text {line,crit }}\right)$ and a supercritical filament (with $\left\langle M_{\text {line }}\right\rangle \sim 30 M_{\odot} / \mathrm{pc}>M_{\text {line,crit }}$ ) in the IC 5146 and Taurus ${ }^{2}$ clouds, respectively.

The column density/line mass longitudinal profiles used in this paper (see Figs. 2b, 5b, and 6b) have an effective 18'.'2-beam

\footnotetext{
2 Here $\left\langle M_{\text {line }}\right\rangle$ was obtained by multiplying the mean backgroundsubtracted column density by a characteristic $0.1 \mathrm{pc}$ width, whereas Palmeirim et al. (2013) obtained a value of $54 M_{\odot} / \mathrm{pc}$ by integration over the full radial column density profile of the filament.
} 

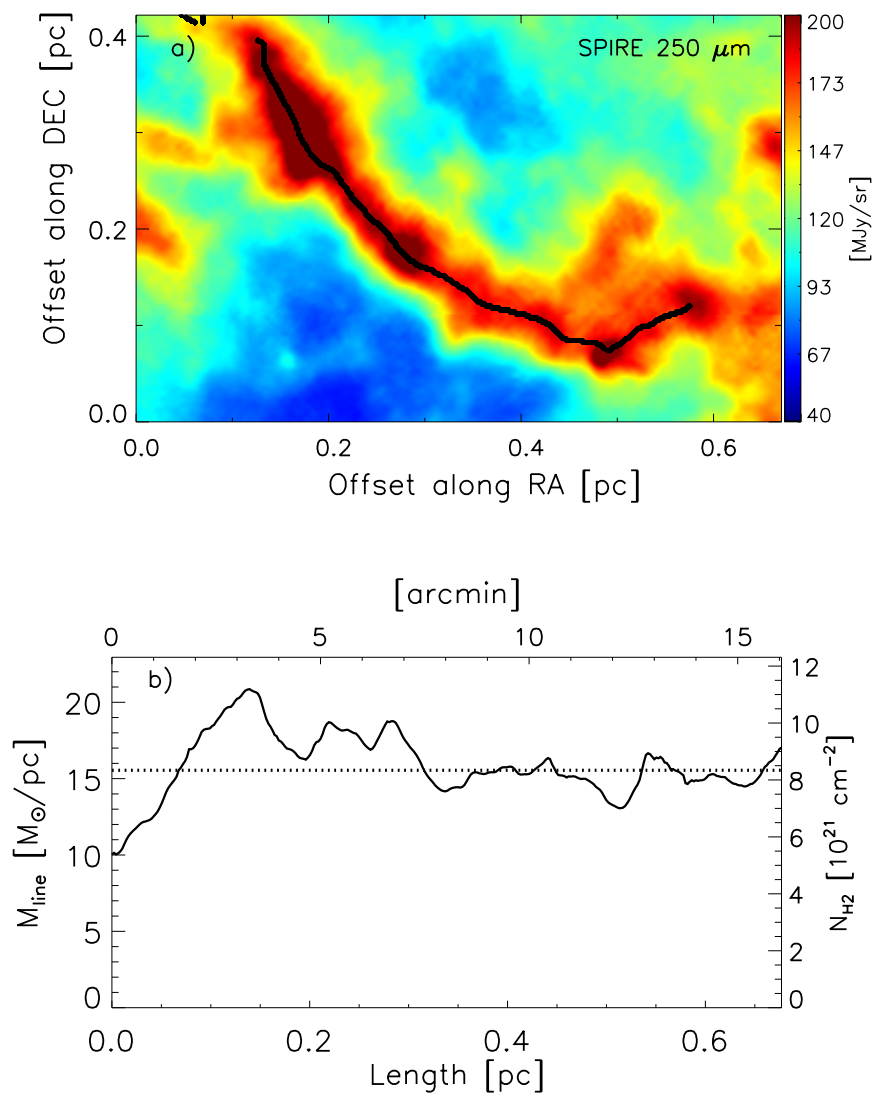

Fig. 2. Close-up view of the sub-region indicated by the black dashed rectangle in Fig. 1 showing a) an example of a nearly critical filament in the Pipe cloud at an adopted distance $d=145 \mathrm{pc}$ and b) the linemass/column density fluctuations along the long axis of the same filament, both at the 18 ". 2 (HPBW) resolution of the SPIRE $250 \mu \mathrm{m}$ data. The black curve in a) shows the crest of the filament as traced by the DisPerSE algorithm (cf. Sousbie et al. 2011). In b) the right ordinate axis shows the net $\mathrm{H}_{2}$-column density fluctuations along the filament. Equivalent line-mass values (assuming a characteristic filament width of $0.1 \mathrm{pc}$ ) are indicated on the left ordinate axis. A constant background column density level was subtracted before deriving the line mass fluctuations. The dotted horizontal line marks the mean line mass of the filament, $\left\langle M_{\text {line }}\right\rangle \sim 15 M_{\odot} / \mathrm{pc}$, which is only slightly lower than critical line mass of $16.3 M_{\odot} / \mathrm{pc}$ for an isothermal cylinder at $10 \mathrm{~K}$. The median relative amplitude of the line-mass fluctuations is $\frac{\mid M_{\text {line }}-\left\langle M_{\text {line }}\right\rangle}{\left\langle M_{\text {line }}\right\rangle} \sim 0.07$, within the linear perturbation regime. The maximum relative amplitude is $\sim 0.3 \ll 1$. (This figure is available in color in electronic form.)

resolution. The pixel resolution of the corresponding data is $6{ }^{\prime \prime} 0$, close to the Nyquist sampling limit $\left(\lambda / 2 D \sim 77^{\prime \prime} 4\right)$ of SPIRE $250 \mu \mathrm{m}$ observations where $D=3.5 \mathrm{~m}$ is the diameter of the primary mirror of the Herschel telescope. When deriving physical properties from the power spectra of individual filaments, we took special care to exclude angular frequencies higher than the Nyquist critical frequency $D / \lambda \sim 1 /\left(2 \times 7.4^{\prime \prime}\right) \sim$ $0.068 \operatorname{arcsec}^{-1} \sim 4.0 \operatorname{arcmin}^{-1}$.

\section{Power spectrum analysis of the filaments}

The normalized power spectrum $P(s)$ of a $1 \mathrm{D}$ spatially-varying field $I(l)$ is proportional to the square of the Fourier amplitude of the signal, and mathematically the relationship in the $1 \mathrm{D}$ case is

$P(s)=\frac{1}{L}|\tilde{I}(s)|^{2}$,

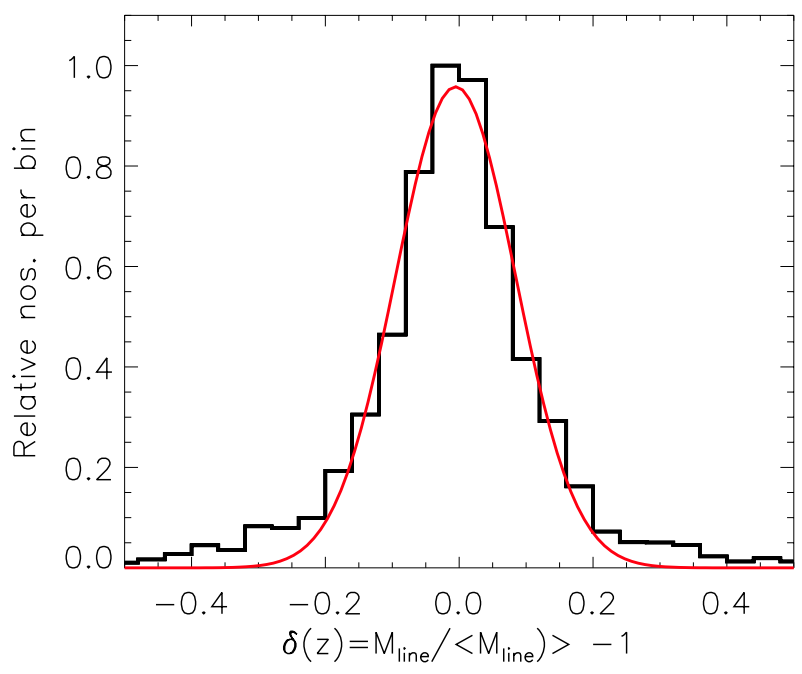

Fig. 3. Normalized histogram showing the probability distribution of relative line-mass fluctuations along the long axes of the subset of 67 filaments, which are entirely subcritical. The red curve shows the Gaussian best fit to the observed distribution, which has a mean of $\sim 0$ and a standard deviation of $\sim 0.09$. (This figure is available in color in electronic form.)

where $s$ denotes spatial frequency (and $\bar{s}$ denotes angular frequency), $\tilde{I}(s)=\int I(l) \mathrm{e}^{-2 \mathrm{i} \pi s l} \mathrm{~d} l$ is the Fourier transform of $I(l)$, and $L=\int \mathrm{d} l$ is the total length over which the signal is measured. In the present case, it is convenient to take $I(l)$ as the field of line-mass fluctuations (in $M_{\odot} / \mathrm{pc}$ ) along a given filament and to express the offset along the filament crest, $l$, in units of pc. Then, the normalized power spectrum $P(s)$ has units of $M_{\odot}^{2} / \mathrm{pc}$.

The observed signal after convolution with the telescope beam, $B$, is given by $I_{\text {obs }}(l)=I_{\text {true }}(l) * B+N(l)$, where $N(l)$ is a noise term arising from small-scale fluctuations primarily due to instrument noise. The Fourier transform of $I_{\mathrm{obs}}(l)$ is $\tilde{I}_{\text {obs }}(s)=\tilde{I}_{\text {true }}(s) \times \tilde{B}(s)+\tilde{N}(s)$. Using Eq. (1) and the previous relation, it can be seen that the total power spectrum observed along the filament axis is composed of the true power spectrum, $P_{\text {true }}(s)$, due to line-mass variations along the filament modified by the power spectrum of the beam, $\gamma_{\text {beam }}(s)$, plus a white noise power spectrum ${ }^{3}$ term, $P_{\mathrm{N}}(s)$, and can be expressed as

$P_{\text {obs }}(s)=P_{\text {true }}(s) \times \gamma_{\text {beam }}(s)+P_{\mathrm{N}}(s)$.

In deriving Eq. (2), we assumed that the noise and the filament line-mass fluctuations are completely uncorrelated, resulting in a vanishing cross-power spectrum term. To estimate the amplitude of $P_{\mathrm{N}}(s)$, we computed the median of the power spectrum in a narrow band of angular frequencies, $3.9 \operatorname{arcmin}^{-1} \leq$ $\bar{s} \leq 4.2 \operatorname{arcmin}^{-1}$, centered about the Nyquist angular frequency of $4.0 \mathrm{arcmin}^{-1}$.

In practice, to recover $P_{\text {true }}(s)$, we first computed $P_{\text {obs }}(s)$ directly from the data, then subtracted a constant noise power

3 The SPIRE bolometers have a measured temporal stability $\gtrsim 250 \mathrm{~s}$, which for Herschel parallel-mode observations taken at a scanning speed of $60^{\prime \prime} \mathrm{s}^{-1}$ translates to a $1 / f$ noise "knee" at spatial frequencies corresponding to angular scales larger than $\sim 4^{\circ}$ (see Fig. 2 of Pascale et al. 2011). The angular scale of the longest filament considered here $\left(\sim 2^{\circ}\right)$ is significantly smaller than both the angular scale of the $1 / f$ knee and the maximum scan length of the corresponding scan-map observations. We can therefore safely assume that the instrumental noise behaves as white noise over the entire range of spatial frequencies considered in the paper. 

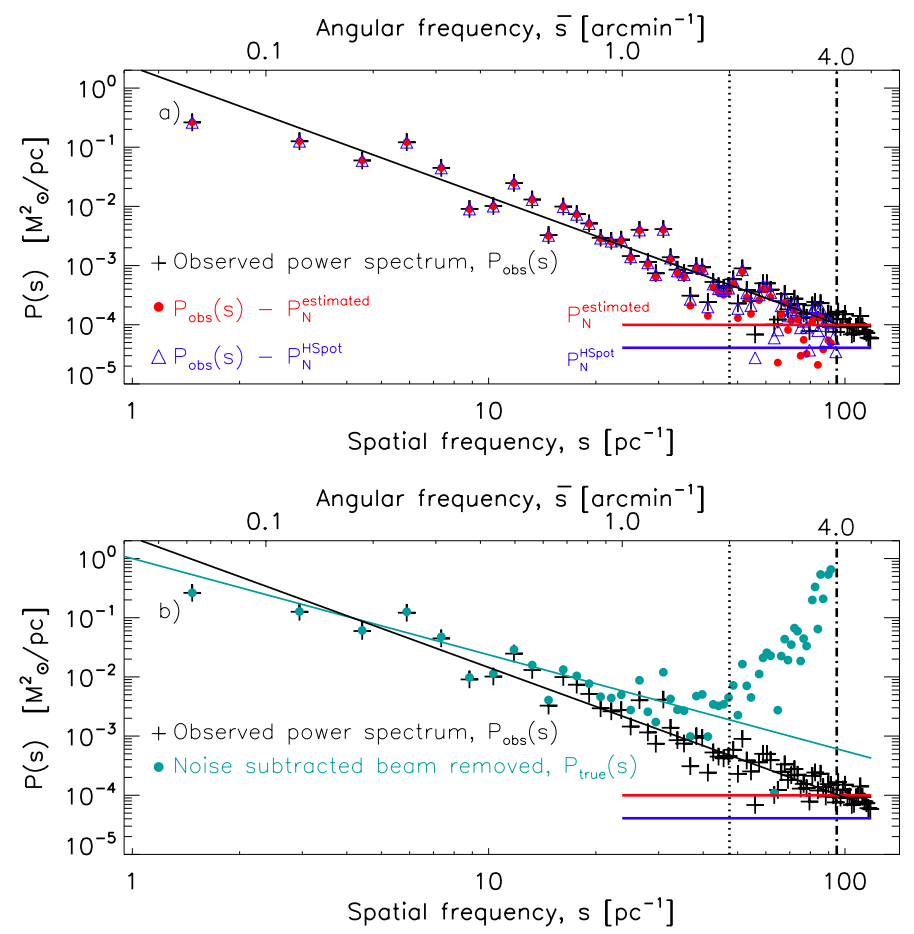

Fig. 4. Power spectrum of the line-mass fluctuations observed along the Pipe filament shown in Fig. 2 as a function of spatial frequency $s$ (bottom $x$-axis) or angular frequency $\bar{s}$ (top $x$-axis). In both panels, the black plus symbols show the observed power spectrum, $P_{\text {obs }}(s)$. In panel a), the red dots show the power spectrum, $P_{\mathrm{obs}}(s)-P_{\mathrm{N}}$, obtained after subtracting a white noise power spectrum level $P_{\mathrm{N}}^{\text {stimated }} \sim 1 \times 10^{-4} M_{\odot}{ }^{2} / \mathrm{pc}$, marked by the horizontal red line and estimated from the median value of $P_{\mathrm{obs}}(s)$ in the 3.9-4.2 $\operatorname{arcmin}^{-1}$ angular frequency range; the blue triangles show a similar power spectrum after subtracting the instrument noise power spectrum level $P_{\mathrm{N}}^{\mathrm{HSpot}} \sim 4.0 \times 10^{-5} M_{\odot}^{2} / \mathrm{pc}$, marked by the horizontal blue line and corresponding to the instrument noise level $1 \sigma \sim 1 \mathrm{MJy} / \mathrm{sr}$ in our SPIRE $250 \mu \mathrm{m}$ maps according to $\mathrm{HSpot}^{3}$. In panel $b$ ), the cyan dots show the noise-subtracted and beam-corrected power spectrum, $P_{\text {true }}(s)=\left(P_{\text {obs }}(s)-P_{\mathrm{N}}\right) / \gamma_{\text {beam }}$. The vertical dotted line in both panels marks the FWHM of the beam power spectrum at $250 \mu \mathrm{m}$ ( $\bar{s} \approx 2 \operatorname{arcmin}^{-1}$; see Fig. A.1), which is also the highest frequency data point used to fit a power-law function. The vertical dot-dashed line is the Nyquist angular frequency $(\lambda / 2 D)$ for SPIRE $250 \mu \mathrm{m}$ data. The powerlaw fits to the power spectra $P_{\text {obs }}(s)$ and $P_{\text {true }}(s)$ have logarithmic slopes $\alpha_{\text {obs }}=-2.1 \pm 0.2$ and $\alpha_{\text {true }}=-1.6 \pm 0.2$, respectively. Considering only angular frequencies up to $\bar{s}=1.5 \mathrm{arcmin}^{-1}$, the best power-law fit to $P_{\text {true }}(s)$ becomes $\alpha_{\text {true }}=-1.7 \pm 0.3$. (This figure is available in color in electronic form.)

level, and finally corrected the result for the beam convolution effect by dividing by $\gamma_{\text {beam }}(s)$. Within the relevant range of angular frequencies $(\bar{s}<F W H M$ of the beam power spectrum $\sim 2 \operatorname{arcmin}^{-1}$ ), both $P_{\text {obs }}(s)$ and $P_{\text {true }}(s)$ can be approximated and fitted by a power-law function,

$P_{\text {obs/true }}(s) \approx P\left(s_{0}\right)\left(s / s_{0}\right)^{\alpha_{\text {obs/true }},}$

where $P\left(s_{0}\right)$ is the normalizing amplitude of the power spectrum at spatial frequency $s_{0}$ and $\alpha$ is the power-law index of the fitted power spectrum.

The results of such a power spectrum analysis are shown in Fig. 4 for the marginally critical filament of Fig. 2. The plus symbols in Fig. 4 correspond to the observed power spectrum of the example filament, $P_{\text {obs }}(s)$, prior to noise subtraction and beam correction. The upper panel of Fig. 4 explicitly shows the effect of noise level subtraction. The horizontal red line marks
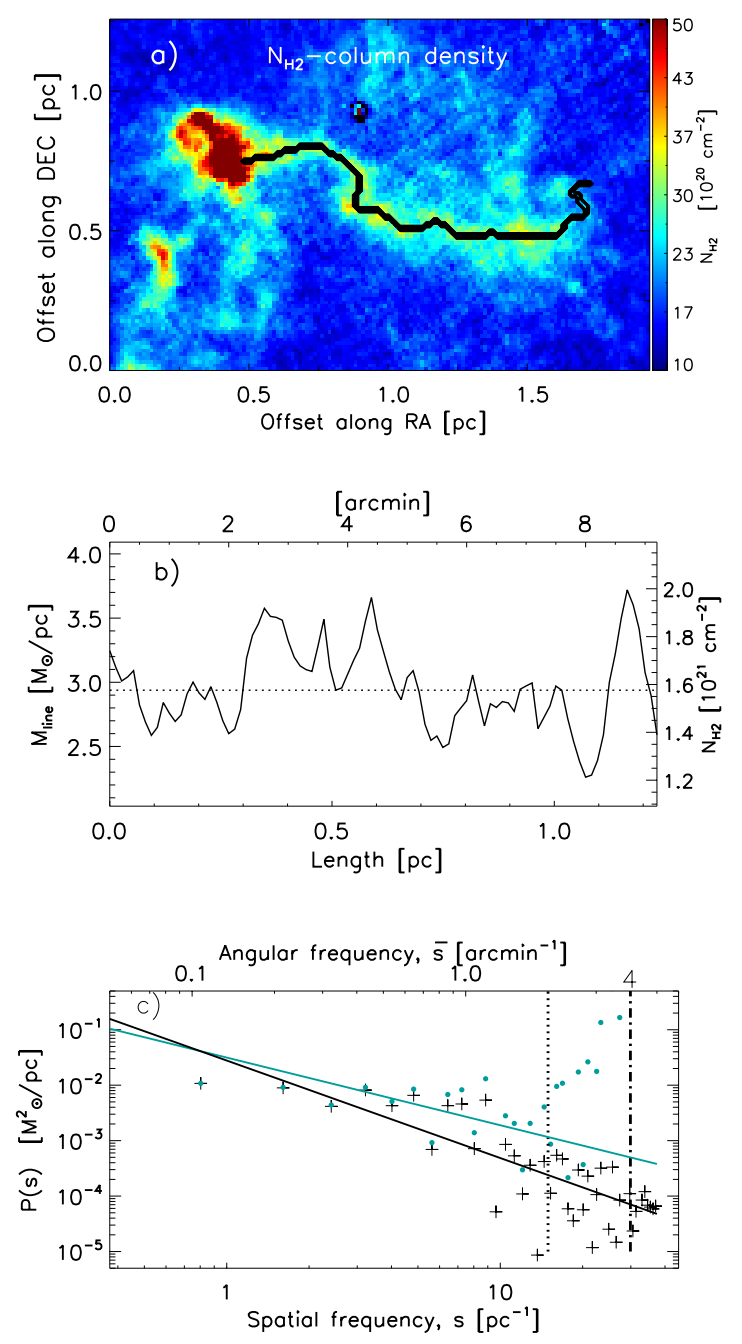

Fig. 5. Panels a) and b) are similar to Figs. $2 \mathrm{a}$ and b, but for an example of a thermally subcritical filament in the IC 5146 molecular cloud $(d \sim$ $460 \mathrm{pc})$. The mean line mass of the filament is $\left\langle M_{\text {line }}\right\rangle \sim 3 M_{\odot} / \mathrm{pc} \ll$ $M_{\text {line,crit }}$, as shown by the dotted horizontal line in panel (b). The median relative amplitude of the line-mass fluctuations is $\frac{\left|M_{\text {line }}-\left\langle M_{\text {line }}\right\rangle\right|}{\left\langle M_{\text {line }}\right\rangle} \sim 0.06$, within the linear perturbation regime. Panel c) is similar to Fig. 4 but for the IC 5146 filament. The power-law fits to the power spectra $P_{\text {obs }}(s)$ and $P_{\text {true }}(s)$ have logarithmic slopes $-1.8 \pm 0.2$ and $-1.2 \pm 0.4$, respectively. Considering only angular frequencies up to $\bar{s}=1.5 \mathrm{arcmin}^{-1}$, the best power-law fit to $P_{\text {true }}(s)$ has a slope $\alpha_{\text {true }}=-1.1 \pm 0.3$. (This figure is available in color in electronic form.)

the estimated power level due to instrument noise, $P_{\mathrm{N}}^{\text {estimated }}=$ $1 \times 10^{-4} M_{\odot}^{2} / \mathrm{pc}$, derived following the above recipe. For comparison, the blue horizontal line shows the noise power spectrum level $\left(P_{\mathrm{N}}^{\mathrm{HSpot}}\right)$ corresponding to the root mean square $(\mathrm{rms})$ instrument noise level (i.e., $1 \sigma \sim 1 \mathrm{MJy} / \mathrm{sr}$ or $\sim 10 \mathrm{mJy} / \mathrm{beam}$ ) expected for SPIRE $250 \mu \mathrm{m}$ data corresponding to two orthogonal scans taken at $60^{\prime \prime} \mathrm{s}^{-1}$ in parallel-mode observations according to the Herschel Observation Planning Tool (HSpot $)^{4}$. The noise power spectrum level $P_{\mathrm{N}}^{\mathrm{HSpot}}$ was determined using Parseval's theorem which relates the variance of the instrumental noise to the integral of its power spectrum: $\sigma_{\text {Mline,HSpot }}^{2}=$ $\int_{-s_{\max }}^{s_{\max }} P_{\mathrm{N}}^{\mathrm{HSpot}} \mathrm{d} s=2 s_{\max } \times P_{\mathrm{N}}^{\mathrm{HSpot}}$, where the integral is taken over the relevant spatial frequency range given the maximum angular

\footnotetext{
4 http://herschel.esac.esa.int/Docs/HSPOT/html/ hspot-help.html
} 

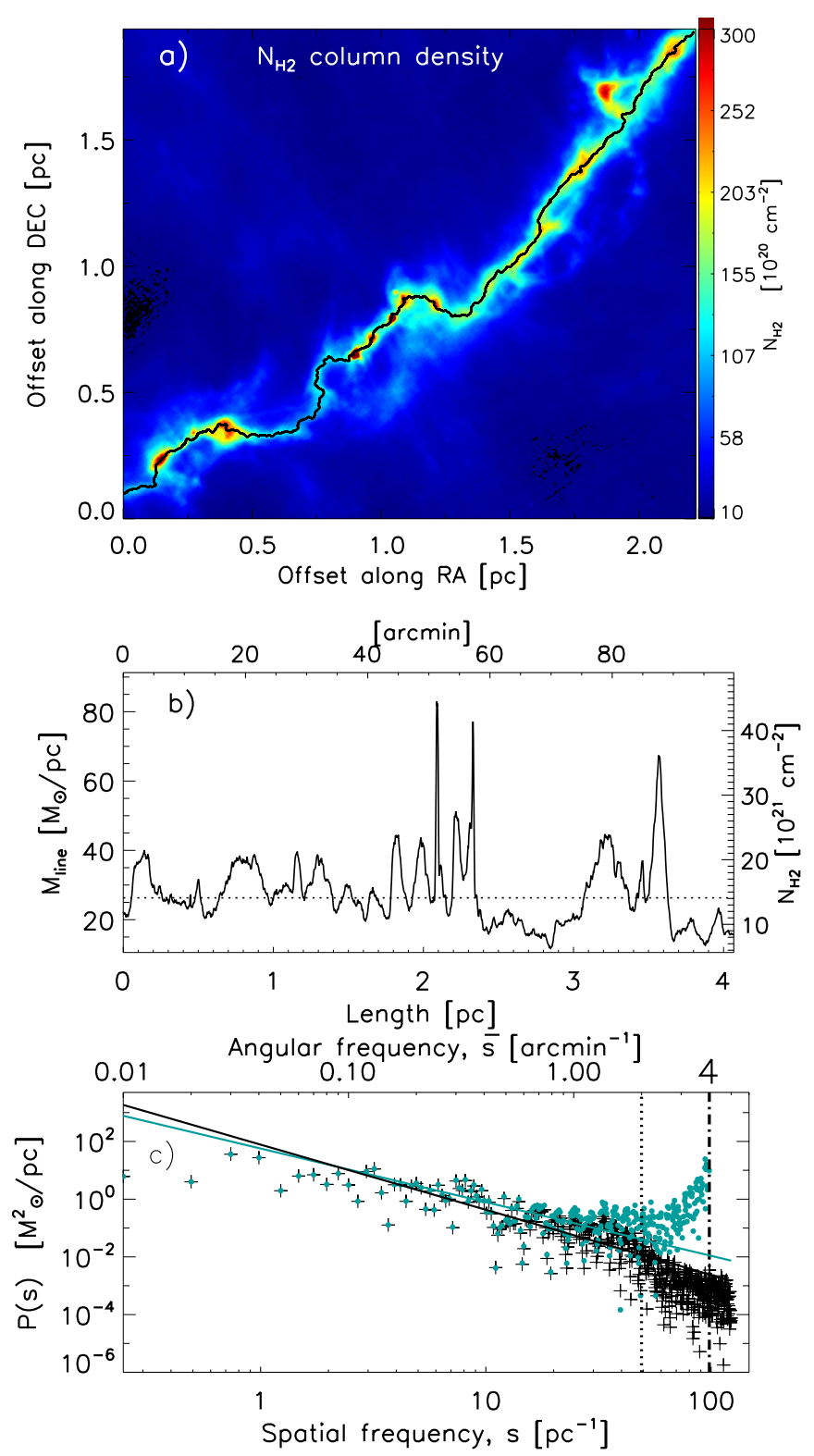

Fig. 6. Similar to Fig. 5, but for the thermally supercritical filament B211/B213 in the Taurus cloud at $d \sim 140$ pc (cf. Palmeirim et al. 2013). The mean line mass of the filament is $\left\langle M_{\text {line }}\right\rangle \sim 30 M_{\odot} / \mathrm{pc} \gg$ $M_{\text {line,crit }}$, as shown by the dotted horizontal line in panel $\left.\mathbf{b}\right)$. The median relative amplitude of the line-mass fluctuations is $\frac{\left|M_{\text {line }}-\left\langle M_{\text {line }}\right\rangle\right|}{\left\langle M_{\text {line }}\right\rangle} \sim 0.4$. We note the spike features corresponding to the positions of dense cores along the filament and capturing non-linear density perturbations. In panel c), the power-law fits to the power spectra $P_{\text {obs }}(s)$ and $P_{\text {true }}(s)$ have logarithmic slopes $-2.2 \pm 0.2$ and $-1.9 \pm 0.3$, respectively. The best power-law fit to $P_{\text {true }}(s)$ is unchanged if only angular frequencies up to $\bar{s}=1.5 \mathrm{arcmin}^{-1}$ are considered. (This figure is available in color in electronic form.)

frequency $\bar{s}_{\text {max }}=4.0 \mathrm{arcmin}^{-1}$ sampled by our SPIRE $250 \mu \mathrm{m}$ data. For a $0.1 \mathrm{pc}$ wide filament with a median dust temperature of $13.5 \mathrm{~K}$ as measured along the filament crest in Fig. 2, a $1 \sigma \mathrm{rms}$ level of $\sim 1 \mathrm{MJy} / \mathrm{sr}$ at $250 \mu \mathrm{m}$ corresponds to a standard deviation $\sigma_{\text {Mline,HSpot }} \sim 0.09 M_{\odot} /$ pc in line-mass units. Given the distance $d=145 \mathrm{pc}$ to the filament of Fig. 2, this yields $P_{\mathrm{N}}^{\mathrm{HSpot}}=\sigma_{\text {Mline,HSpot }}^{2} /\left(2 s_{\max }\right) \approx 4.0 \times 10^{-5} M_{\odot}^{2} / \mathrm{pc}$ in the same unit as that used on the $y$-axis of Fig. 4 .
The red dots and overplotted blue triangles in Fig. 4a show the power spectrum data points after subtracting the estimated noise spectrum level $P_{\mathrm{N}}^{\text {estimated }}$ and the expected instrument noise spectrum level $P_{\mathrm{N}}^{\mathrm{HSpot}}$, respectively. It is apparent from Fig. 4a that the departure of the noise-subtracted data points from the observed filament power spectrum is negligible in the angular frequency range $\bar{s}<2 \operatorname{arcmin}^{-1}$ where we fit a powerlaw slope. Therefore, even though our estimated noise spectrum level, $P_{\mathrm{N}}^{\text {estimated }}$, likely overestimates the actual noise spectrum level, the step of noise subtraction has effectively no influence on the estimated power-law slope of the observed power spectrum.

The beam-corrected power spectrum, $P_{\text {true }}(s)$, shown by the cyan solid dots in Fig. 4b was obtained by dividing the noisesubtracted power spectrum by the $250 \mu \mathrm{m}$ SPIRE beam power spectrum $\left(\gamma_{\text {beam }}\right)$. The derivation of the SPIRE beam power spectrum is described in Appendix A. The $P_{\text {obs }}(s)$ spectrum has more power at low spatial frequencies $s$ (large scales) and decreases roughly as a power law toward high spatial frequencies until it merges with the noise power spectum level at a spatial frequency close to $s \sim 100 \mathrm{pc}^{-1}$ (i.e., angular frequency $\sim 4.0 \mathrm{arcmin}^{-1}$ ). The beam-corrected power spectrum, $P_{\text {true }}(s)$, has a slightly shallower slope than $P_{\mathrm{obs}}(s)$. At spatial frequencies higher than the FWHM of the beam power spectrum shown by the dotted vertical line at $s \sim 50 \mathrm{pc}^{-1}$ (angular frequency $\bar{s} \sim 2 \operatorname{arcmin}^{-1}$ ) in Fig. $4, P_{\text {true }}(s)$ is overcorrected owing to amplification of residual noise when dividing by the beam power spectrum. This unphysical rise of $P_{\text {true }}(s)$ (see Fig. 4b) at very small angular frequencies is a generic feature of beam-corrected power spectra and is well documented in earlier Herschel studies of cirrus noise (e.g., Martin et al. 2010; Miville-Deschênes et al. 2010). To get around this problem, it is customary to avoid angular frequencies greater than the FWHM of the beam power spectrum, i.e., $\bar{s}>2 \operatorname{arcmin}^{-1}$ at $250 \mu \mathrm{m}$ (cf. Miville-Deschênes et al. 2010), a compromise that we also adopt in this paper. Upon visually inspecting Fig. 4b, there may still be an indication of a slight overcorrection of $P_{\text {true }}(s)$ in the frequency range $1.5 \operatorname{arcmin}^{-1} \lesssim \bar{s} \leq$ $2 \operatorname{arcmin}^{-1}$. Therefore, we also report the results of more conservative power-law fits obtained by fitting $P_{\text {true }}(s)$ only up to $\bar{s}=1.5 \operatorname{arcmin}^{-1}$. Finally, it is common practice to avoid very small spatial frequencies when fitting power spectrum slopes in the case of 2D images because of edge effects or large scanning lengths, for example. In principle, in the case of a filament much smaller than the size of the mapped region, there should be no technical difficulty in reconstructing features at all angular scales with Fourier modes. To be on the safe side, we nevertheless excluded the smallest non-zero angular frequency data point when fitting a power law to the power spectrum of each filament ${ }^{5}$.

For the Pipe filament shown in Fig. 4, the beam-corrected power spectrum has a best-fit slope $\alpha_{\text {true }}=-1.6 \pm 0.2$ and an amplitude $P\left(s_{0}=10 \mathrm{pc}^{-1}\right)=9.5 \times 10^{-2} M_{\odot}{ }^{2} / \mathrm{pc}$. For comparison, the observed power spectrum has a somewhat steeper best-fit slope $\alpha_{\text {obs }}=-2.1 \pm 0.2$ and an amplitude $P\left(s_{0}=10 \mathrm{pc}^{-1}\right)=$ $6 \times 10^{-2} M_{\odot}^{2} / \mathrm{pc}$. Two other examples of filament power spectra are shown in Fig. 5c and Fig. 6c for the IC 5146 subcritical filament of Fig. 5a and the Taurus supercritical filament of Fig. 6a, respectively.

\footnotetext{
5 Including the lowest $s$ data point when fitting the power spectrum slope would change the overall distribution of slopes in Fig. 7 by less than one standard deviation.
} 
A. Roy et al.: Power spectra

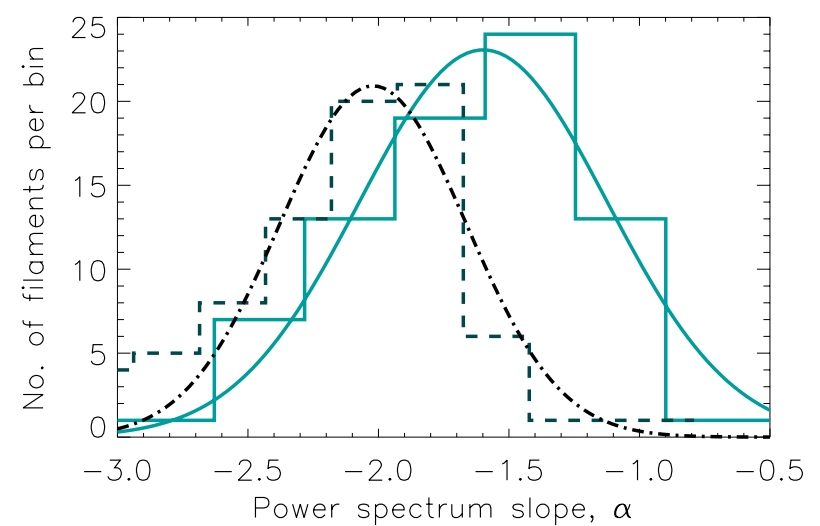

Fig. 7. Distributions of power spectrum slopes measured before beam correction (dashed histogram) and after beam correction (solid histogram) for our selected sample of 80 filaments. Best-fit Gaussian curves to the two observed distributions are overplotted. The two distributions are centered on $\bar{\alpha}_{\text {obs }}=-2.0 \pm 0.2$ and $\bar{\alpha}_{\text {corr }}=-1.6 \pm 0.3$ for the uncorrected and beam-corrected power spectra, respectively. (This figure is available in color in electronic form.)

\subsection{Characteristic power spectrum slope for filaments}

To reach statistically significant conclusions on the typical power spectrum slope, we considered a sample of 80 subcritical (or marginally supercritical) filaments belonging to three distinct nearby molecular clouds (see Sect. 3). Since the reliability of the derived power spectrum properties also depends on the dynamic range covered in spatial frequency space, we excluded from our present analysis filaments that were smaller than 5.5 in the plane of sky. The resulting distribution of power spectrum slopes is shown in Fig. 7. The two histograms shown by the dashed and solid lines represent the distributions obtained for the observed $\left[P_{\text {obs }}(s)\right]$ and beam-corrected $\left[P_{\text {true }}(s)\right]$ filament power spectra, respectively. Both histograms are well fitted by Gaussian distributions (see Fig. 7).

The mean power-law index $\bar{\alpha}_{\text {obs }}=-2.0 \pm 0.2$ measured for the observed power spectra is slightly steeper than the mean power-law index $\bar{\alpha}_{\text {corr }}=-1.6 \pm 0.3$ obtained at $\bar{s}<2 \operatorname{arcmin}^{-1}$ after correcting for the beam convolution effect. Fitting $P_{\text {true }}(s)$ with a power law over the more conservative range of angular frequencies $\bar{s} \leq 1.5 \mathrm{arcmin}^{-1}$ yields essentially the same index within errors, $\bar{\alpha}_{\text {corr }}=-1.8 \pm 0.5$. The distribution of beamcorrected power spectrum slopes has a larger dispersion than the distribution of uncorrected slopes, due to the propagation of uncertainties resulting from the amplification of residual noise at small scales.

\subsection{Correlation between $P\left(s_{0}\right)$ and mean column density}

Figure 8 shows a positive correlation between the amplitude of the line-mass fluctuations, expressed by $P^{1 / 2}\left(s_{0}\right)$, and the mean column density of the filaments. In other words, filaments with higher mean column densities tend to have higher power spectrum amplitudes, i.e., stronger column density fluctuations. The red straight line in Fig. 8 shows the best-fit power law to the data points, which has an index of $1.4 \pm 0.1$. Physically, this empirical relation means that the rms fluctuations of the perturbative modes inside a filament increase with the mean line mass of the filament. A similar empirical correlation with a somewhat different power-law index $\left(P_{\text {cirrus }} \propto\left\langle S_{v}\right\rangle^{3}\right)$ exists between the 2D power spectrum amplitude of far-infrared cirrus $\left(P_{\text {cirrus }}\right)$ and

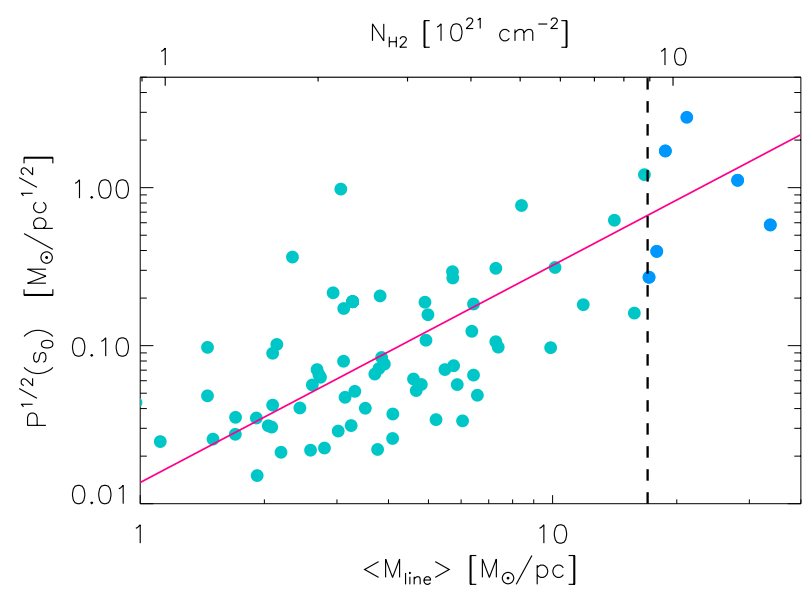

Fig. 8. Square root of filament power spectrum amplitude at $s_{0}=10 \mathrm{pc}^{-1}$ versus mean line mass of each filament. The solid line shows the bestfit power-law $P^{1 / 2}\left(s_{0}\right) \propto M_{\text {line }}^{1.4+0.1}$. The corresponding mean $N_{\mathrm{H}_{2}}$-column density level is shown by the upper coordinate axis. The vertical dashed line separates subcritical filaments (cyan filled circles on the left) from supercritical filaments (blue filled circles on the right). (This figure is available in color in electronic form.)

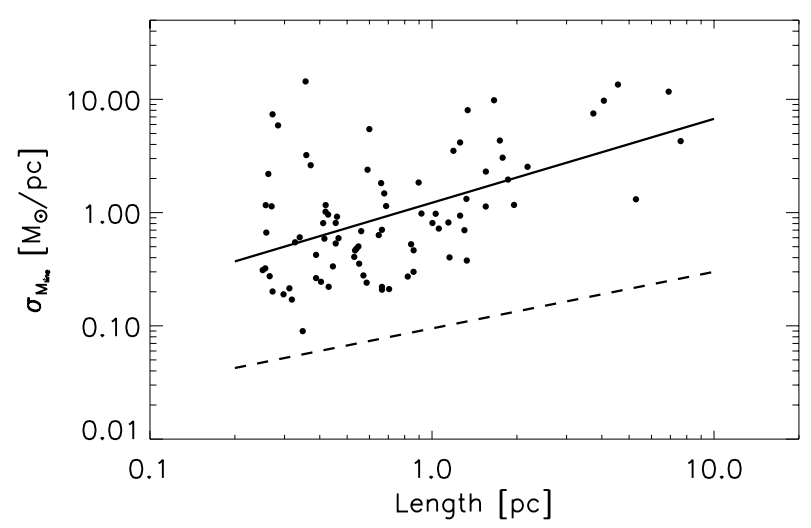

Fig. 9. Dispersion of line-mass fluctuations $\sigma_{M_{\text {line }}}$ versus projected length $L$ for the filaments in our sample. The solid line shows the best-fit power-law $\sigma_{M_{\text {line }}} \propto L^{0.7}$ to the data points. For comparison, the dashed line shows a power-law scaling equivalent to the well-known linewidthsize relation in molecular clouds $\sigma_{\mathrm{v}} \propto L^{0.5}$ (cf. Larson 1981), with an arbitrary normalization.

the average surface brightness $\left\langle S_{v}\right\rangle$ in far-infrared images of the sky (Gautier et al. 1992).

\subsection{Line-mass dispersion versus filament length}

Figure 9 shows that the dispersion of line-mass fluctuations along the long axis of each filament, $\sigma_{M_{\text {line }}}$, is correlated with the physical length $L$ of the filament. The best-fit power-law relation between $\sigma_{M_{\text {line }}}$ and $L$ is $\sigma_{M_{\text {line }}} \propto L^{0.7}$, which is reminiscent of the well-known power-law scaling between internal velocity dispersion and region size in molecular clouds $\left(\sigma_{\mathrm{v}} \propto L^{0.5}\right)$ originally found by Larson (1981). Such a similarity is not surprising since, in the subsonic turbulence regime which approximately holds for subcritical or marginally subcritical filaments (Arzoumanian et al. 2013), density fluctuations are expected to be directly proportional to velocity fluctuations. 


\section{Concluding remarks}

Our 1D power spectrum analysis along the axis of 80 nearby Herschel filaments shows that the longitudinal line-mass fluctuations along these filaments have a characteristic 1D power spectrum slope $\bar{\alpha}_{\text {corr }}=-1.6 \pm 0.3$. This result is the first observational confirmation that the density/line-mass fluctuations along interstellar filaments have a characteristic power spectrum slope. The slope we find is remarkably close to the fiducial value $\alpha_{\text {theory }}=-1.5$ adopted by Inutsuka (2001) in his attempt to explain the origin of Salpeter-like power-law tail of the CMF/IMF at the high-mass end $\left(\mathrm{d} N / \mathrm{d} M \propto M^{-2.5}\right)$. Repeating Inutsuka's analysis with the observationally derived power spectrum slope $\bar{\alpha}_{\text {corr }}=-1.6$ found in the present study ${ }^{6}$ would lead to a CMF approaching $\mathrm{d} N / \mathrm{d} M \propto M^{-2.4}$ at the high-mass end after a few free-fall times, which is even closer to a Salpeter mass function $\left(\mathrm{d} N / \mathrm{d} M \propto M^{-2.35}\right)$.

Interestingly, the measured power spectrum of density fluctuations along interstellar filaments shows a striking similarity to the energy spectrum or velocity power spectrum of incompressible hydrodynamical turbulence in $1 \mathrm{D}$, i.e., $E(s) \propto P_{\mathrm{v}}^{\text {turb }}(s) \propto$ $s^{-5 / 3}$ (Kolmogorov 1941) ${ }^{7}$. For a weakly compressible flow, as long as self-gravity does not dominate, the continuity equation implies a direct proportionality between the Fourier modes of small density fluctuations and the modes of subsonic velocity perturbations. Our measured power spectrum slope for the density fluctuations $\left(\bar{\alpha}_{\text {corr }}=-1.6 \pm 0.3\right)$ is thus consistent with the fact that subcritical and marginally supercritical filaments - like the filaments considered in the present study - are observed to have subsonic or at most transonic turbulent velocity dispersions (Hacar \& Tafalla 2011; Arzoumanian et al. 2013).

The origin of the characteristic power spectrum slope of filaments may have a close connection with the process generating cirrus fluctuations of far-infrared surface brightness in the interstellar medium. The statistical analysis of Galactic cirrus fluctuations in IRAS 2D images by Gautier et al. (1992) has suggested a plausible role of interstellar turbulence in shaping these fluctuations. The power spectrum of cirrus fluctuations obtained from Roy et al. (2010) and recent Herschel images (Martin et al. 2010; Miville-Deschênes et al. 2010) has a power-law slope of -2.7 , very similar to the slope of $-8 / 3 \approx-2.67$ expected for the velocity power spectrum of Kolmogorov turbulence in $2 \mathrm{D}$. This $2 \mathrm{D}$ slope translates to a power spectrum slope of $-5 / 3 \approx-1.67$ in $1 \mathrm{D}^{8}$.

Our Herschel results support the following speculative picture. Filamentary structures form in the cold interstellar medium as a result of a combination of compression and shear in large-scale magneto-hydrodynamic flows (Padoan et al. 2001; Balsara et al. 2001; Hennebelle 2013; Inutsuka et al. 2015). Supersonic turbulent motions dissipate quickly within dense filaments, forming more stable and coherent structures, and leav-

\footnotetext{
${ }_{6}$ Here we adopt the mean beam-corrected value $\bar{\alpha}_{\text {corr }}=1.6$ derived in Sect. 5.1 as our best estimate of the power spectrum slope. Using the prescription of Inutsuka (2001) with the mean power-law index $\bar{\alpha}_{\text {obs }}=$ -2.0 measured before beam correction (see Fig. 7) instead would lead to a CMF approaching $\mathrm{d} N / \mathrm{d} M \propto M^{-2.2}$ after a few free-fall times.

7 Given the uncertainties associated with beam correction (see Sect. 5), the mean beam-corrected index $\bar{\alpha}_{\text {corr }}=-1.6 \pm 0.3$ is marginally consistent with both the energy spectrum $E(s) \propto P_{\mathrm{v}}^{\text {turb }}(s) \propto s^{-2}$ (cf. Kritsuk et al. 2007) and the 1D density power spectrum (cf. Federrath et al. 2009) expected for supersonic compressible turbulence.

8 The general relation between the Kolmogorov energy spectrum $E(s) \propto s^{-5 / 3}$ and the velocity power spectrum in $n$-D space is $E(s) \propto$ $s^{n-1} P_{\mathrm{v}}(s)$.
}

ing an imprint of subsonic fluctuations along the filaments. Molecular line observations of the inner parts of subcritical or marginally supercritical filaments do show subsonic or transonic velocity dispersions (Hacar \& Tafalla 2011; Arzoumanian et al. 2013), supporting the view that the dissipation of turbulence may play a role in the formation and evolution of these structures. The density fluctuations along supercritical filaments are Jeansunstable and grow by local gravitational instability, generating a distribution of prestellar cores.

The observational verification of the existence of a characteristic power spectrum slope for line-mass fluctuations along interstellar filaments has therefore opened an interesting avenue for understanding an important aspect of the origin of the prestellar CMF. Our findings suggest that an initial spectrum of density perturbations seeded by interstellar turbulence may be a prerequisite for generating a Salpeter-like CMF at the high-mass end. The present study combined with previous Herschel results therefore provides insight into the possible origin of two distinct features of the CMF: the thermal gravitational fragmentation scale of filaments may set the peak of the lognormal base of the prestellar CMF (e.g., André et al. 2014), while the evolutionary characteristics of the underlying line-mass perturbations may help to set the power-law slope of the CMF/IMF at the high mass end (Inutsuka 2001 and this paper). A final caveat should be mentioned, however. There is mounting evidence that true high-mass analogs to low-mass prestellar cores may not exist (e.g., Motte et al. 2007) and that massive protostars may acquire the bulk of their final mass from much larger scales than a single prestellar core (e.g., Peretto et al. 2013). Further work will be needed to evaluate the relative importance of initial density fluctuations and large-scale accretion in generating the highmass end of the IMF'.

Acknowledgements. We are thankful to Prof. Shu-Ichiro Inutsuka for stimulating and enlightening discussions on filaments. We also acknowledge insightful discussions with Patrick Hennebelle and Gilles Chabrier about the origin of the CMF/IMF and the Press-Schechter formalism. This work has received support from the European Research Council under the European Union's Seventh Framework Programme (ERC Advanced Grant Agreement No. 291294 - "ORISTARS") and from the French National Research Agency (Grant No. ANR-11-BS56-0010 - "STARFICH"). SPIRE has been developed by a consortium of institutes led by Cardiff Univ. (UK) and including Univ. Lethbridge (Canada); NAOC (China); CEA, LAM (France); IFSI, Univ. Padua (Italy); IAC (Spain); Stockholm Observatory (Sweden); Imperial College London, RAL, UCL-MSSL, UKATC, Univ. Sussex (UK); Caltech, JPL, NHSC, Univ. Colorado (USA). This development has been supported by national funding agencies: CSA (Canada); NAOC (China); CEA, CNES, CNRS (France); ASI (Italy); MCINN (Spain); SNSB (Sweden); STFC (UK); and NASA (USA). PACS has been developed by a consortium of institutes led by MPE (Germany) and including UVIE (Austria); KUL, CSL, IMEC (Belgium); CEA, OAMP (France); MPIA (Germany); IFSI, OAP/AOT, OAA/CAISMI, LENS, SISSA (Italy); IAC (Spain). This development has been supported by the funding agencies BMVIT (Austria), ESA-PRODEX (Belgium), CEA/CNES (France), DLR (Germany), ASI (Italy), and CICT/MCT (Spain).

\section{References}

Alves, J., Lombardi, M., \& Lada, C. J. 2007, A\&A, 462, L17

André, P., Men'shchikov, A., Bontemps, S., et al. 2010, A\&A, 518, L102

André, P., Di Francesco, J., Ward-Thompson, D., et al. 2014, in Protostars and Planets VI, ed. H. Beuther (Tuscon, AZ: Univ. of Arizona press), 27 Arzoumanian, D., André, P., Didelon, P., et al. 2011, A\&A, 529, L6 Arzoumanian, D., André, P., Peretto, N., \& Könyves, V. 2013, A\&A, 553, A119 Balsara, D., Ward-Thompson, D., \& Crutcher, R. M. 2001, MNRAS, 327, 715

9 Another, more theoretical issue is that, for technical reasons related to the Press-Schechter formalism, the model discussed by Inutsuka (2001) may, strictly speaking, not be a model for the mass function of (prestellar) cores, but a model for the mass function of groups of cores (P. Hennebelle, priv. comm.). 


\section{A. Roy et al.: Power spectra}

Bernard, J.-P., Paradis, D., Marshall, D. J, et al. 2010, A\&A, 518, L88 Chabrier, G., Hennebelle, P., \& Charlot, S. 2014, ApJ, 796, 75

Elias, J. H. 1978, ApJ, 224, 857

Federrath, C., Klessen, R. S., \& Schmidt, W. 2009, ApJ, 692, 364

Federrath, C., Roman-Duval, J., Klessen, R. S., Schmidt, W., \& Mac Low, M.-M. 2010, A\&A, 512, A81

Gautier, III, T. N., Boulanger, F., Perault, M., \& Puget, J. L. 1992, AJ, 103, 1313

Griffin, M. J., Abergel, A., Abreu, A., et al. 2010, A\&A, 518, L3

Hacar, A., \& Tafalla, M. 2011, A\&A, 533, A34

Heiderman, A., Evans, II, N. J., Allen, L. E., Huard, T., \& Heyer, M. 2010, ApJ, 723,1019

Hennebelle, P. 2013, A\&A, 556, A153

Hennebelle, P., \& Chabrier, G. 2008, ApJ, 684, 395

Hildebrand, R. H. 1983, QJRAS, 24, 267

Hill, T., Motte, F., Didelon, P., et al. 2011, A\&A, 533, A94

Hopkins, P. F. 2012, MNRAS, 423, 2037

Inutsuka, S.-I. 2001, ApJ, 559, L149

Inutsuka, S.-I., \& Miyama, S. M. 1997, ApJ, 480, 681

Inutsuka, S.-I., Inoue, T., Iwasaki, K., \& Hosokawa, T. 2015, A\&A, 580, A49

Jedamzik, K. 1995, ApJ, 448,

Kolmogorov, A. 1941, Akademiia Nauk SSSR Doklady, 30, 301

Könyves, V., André, P., Men'shchikov, A., et al. 2010, A\&A, 518, L106

Könyves, V., André, P., Men'shchikov, A., et al. 2015, A\&A, 584, A91

Kritsuk, A. G., Norman, M. L., Padoan, P., \& Wagner, R. 2007, ApJ, 665, 416

Lada, C. J., Alves, J., \& Lada, E. A. 1999, ApJ, 512, 250
Lada, C. J., Lombardi, M., \& Alves, J. F. 2010, ApJ, 724, 687

Larson, R. B. 1981, MNRAS, 194, 809

Larson, R. B. 1985, MNRAS, 214, 379

Martin, P. G., Miville-Deschênes, M.-A., Roy, A., et al. 2010, A\&A, 518, L105

Miville-Deschênes, M.-A., Martin, P. G., Abergel, A., et al. 2010, A\&A, 518, L104

Molinari, S., Swinyard, B., Bally, J., et al. 2010, A\&A, 518, L100

Motte, F., André, P., \& Neri, R. 1998, A\&A, 336, 150

Motte, F., Bontemps, S., Schilke, P., et al. 2007, A\&A, 476, 1243

Offner, S. S. R., Clark, P. C., Hennebelle, P., et al. 2014, in Protostars and

Planets VI, ed. H. Beuther (Tuscon, AZ: Univ. of Arizona press), 53

Ostriker, J. 1964, ApJ, 140, 1056

Padoan, P., \& Nordlund, A. 2002, ApJ, 576, 870

Padoan, P., Juvela, M., Goodman, A. A., \& Nordlund, Å. 2001, ApJ, 553, 227

Palmeirim, P., André, P., Kirk, J., et al. 2013, A\&A, 550, A38

Pascale, E., Auld, R., Dariush, A., et al. 2011, MNRAS, 415, 911

Peretto, N., André, P., Könyves, V., et al. 2012, A\&A, 541, A63

Peretto, N., Fuller, G. A., Duarte-Cabral, A., et al. 2013, A\&A, 555, A112

Pilbratt, G. L., Riedinger, J. R., Passvogel, T., et al. 2010, A\&A, 518, L1

Poglitsch, A., Waelkens, C., Geis, N., et al. 2010, A\&A, 518, L2

Press, W. H., \& Schechter, P. 1974, ApJ, 187, 425

Roussel, H. 2013, PASP, 125, 1126

Roy, A., Ade, P. A. R., Bock, J. J., et al. 2010, ApJ, 708, 1611

Roy, A., André, P., Palmeirim, P., et al. 2014, A\&A, 562, A138

Sousbie, T., Pichon, C., \& Kawahara, H. 2011, MNRAS, 414, 384 


\section{Appendix A: Deriving the power spectrum of the SPIRE $250 \mu \mathrm{m}$ beam}

The power spectrum of the SPIRE $250 \mu \mathrm{m}$ beam was derived from an empirical PSF image obtained by the SPIRE ICC from scan map data ${ }^{10}$ of Neptune in four directions. There are two sets of empirical PSFs available, one gridded at the nominal pixel size for each SPIRE passband and the other at a higher pixel resolution of 0.6 . We used the latter product, but the angular extent of the footprint was only about 6.0 , corresponding to an angular frequency scale of $\sim 0.3 \mathrm{arcmin}^{-1}$, insufficient to correct for the beam convolution effect on large scales (i.e., small $\bar{s}$ ). Therefore, to extend the angular frequency range of the beam power spectrum to lower $\bar{s}$ values, we embedded the beam map of Neptune inside a larger map 25'0 on a side, and then padded the pixels outside the central Neptune insert using the best-fit 2D Gaussian approximation to the beam.

The power spectrum of the SPIRE $250 \mu \mathrm{m}$ beam as a function of angular frequency, $\gamma_{\text {beam }}(\bar{s})$, is shown in Fig. A.1. The power spectrum asymptotically converges to unity at small angular frequencies, but rapidly declines toward high angular frequencies (small angular scales). We caution that the SPIRE beam spectrum has a slight suppression of power around $0.2 \operatorname{arcmin}^{-1}<\bar{s}<1.5 \operatorname{arcmin}^{-1}$ compared to a pure Gaussian shape. This problem led Martin et al. (2010) and Miville-Deschênes et al. (2010) to approximate the beam power spectrum using higher-order polynomial corrections to the Gaussian fit. The effective full width at half maximum (FWHM) of the SPIRE $250 \mu \mathrm{m}$ beam power spectrum ${ }^{11}$ is about $2 \operatorname{arcmin}^{-1}$, as shown by the dashed curve in Fig. A.1.

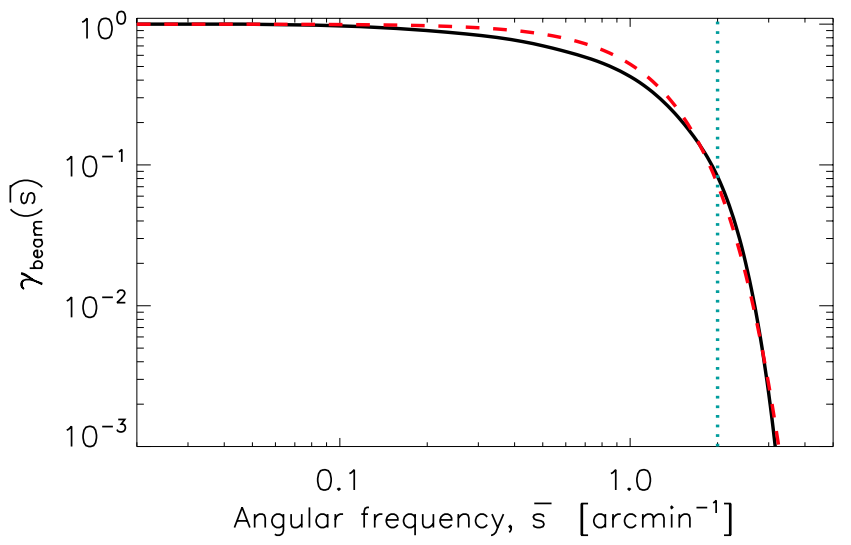

Fig. A.1. Power spectrum of the SPIRE $250 \mu \mathrm{m}$ beam, $\gamma_{\text {beam }}(\bar{s})$ (black solid curve). The dashed curve represents the power spectrum of a Gaussian beam with a FWHM equal to the nominal 18'.2 HPBW of the SPIRE $250 \mu \mathrm{m}$ beam. The dotted vertical line marks the FWHM of the corresponding power spectrum in the angular frequency domain.

\section{Appendix B: Results from high-resolution column density maps}

Figure B.1 shows the power spectrum of the same filament already displayed in Fig. 2, but here derived from a high-resolution

\footnotetext{
10 The reduction of the beam map from scan-map data of Neptune is described at http://herschel.esac.esa.int/Docs/SPIRE/html/ spire_om.html

${ }^{11}$ For a Gaussian beam the beam power spectrum is also Gaussian, and its FWHM $\Gamma$, is related to the half power beam width (HPBW), $\Omega$, by $\Gamma=\sqrt{8} \ln 2 / \pi \Omega$.
}

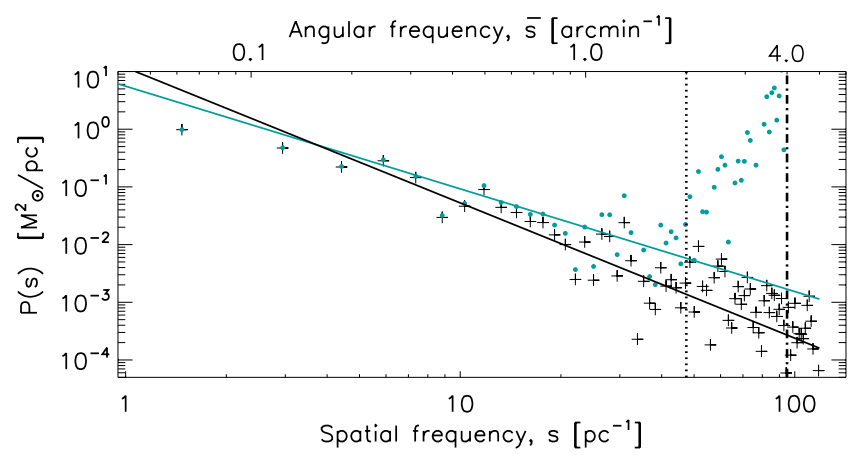

Fig. B.1. Power spectrum of the line-mass fluctuations along the Pipe filament shown in Fig. 2 as measured in the high-resolution $\mathrm{H}_{2}$-column density map resulting from the multi-scale decomposition technique of Palmeirim et al. (2013) Fig. 4. The power-law fits to the power spectra $P_{\text {obs }}(s)$ (black plus symbols) and $P_{\text {true }}(s)$ (cyan filled circles) have logarithmic slopes $\alpha_{\mathrm{obs}}=-2.3 \pm 0.2$ and $\alpha_{\text {corr }}=-1.7 \pm 0.3$, respectively.

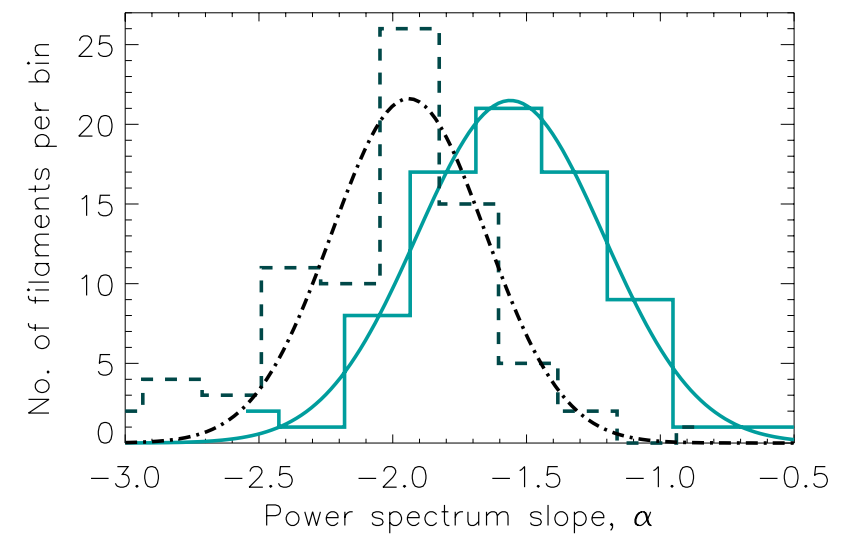

Fig. B.2. Distributions of power spectrum slopes measured in the highresolution $\mathrm{H}_{2}$-column density maps before beam correction (dashed histogram) and after beam correction (solid histogram). Similar to Fig. 7 but based on data from the high-resolution column density maps instead of the modified $250 \mu \mathrm{m}$ maps. Best-fit Gaussian curves to the two observed distributions are overplotted. The two distributions are centered on $\alpha_{\text {obs }}=-1.9 \pm 0.3$ and $\alpha_{\text {corr }}=-1.6 \pm 0.45$ for the uncorrected and beam-corrected power spectra, respectively.

column density map obtained using the multi-scale decomposition scheme of Palmeirim et al. (2013). The power spectrum values at high spatial frequencies has more scatter than the one derived by converting $I_{250}$ to $\mathrm{H}_{2}$-column density (see Fig. 4). This is primarily because the multi-scale decomposition technique introduces fluctuations at small spatial scales which manifest themselves through enhanced scatter in the Fourier modes at high spatial frequencies. The distribution of power spectrum slopes derived from high-resolution column density maps for the whole sample of filaments is shown in Fig. B.2. The mean power spectrum slopes before and after correcting for the beam effect are $\bar{\alpha}_{\text {obs }}=-1.9 \pm 0.3$ and $\bar{\alpha}_{\text {corr }}=-1.6 \pm 0.45$, respectively. Within the quoted errors, these results are indistinguishable from those found in Sect. 5.1 using modified $250 \mu \mathrm{m}$ maps. 